\title{
LITHIUM ABUNDANCE AND SPACIAL DISTRIBUTION OF T TAURI STARS
}

\author{
Jane Gregorio-Hetem and Jacques R.D. Lépine \\ Instituto Astronômico e Geofísico, Universidade de São Paulo \\ CP 9638, 01065 São Paulo, Brasil
}

\begin{abstract}
We determined temperatures and Lithium $6707 \mathrm{~A}$ resonance line equivalent width of a sample of $62 \mathrm{~T}$ Tauri stars. Lithium abundances were then estimated by using a grid of curves of growth. The lithium abundance is shown to decrease with the distance of the stars to the nearest dense core of a molecular cloud. This effect is interpreted as being due to the ages of the stars, the youngest ones being closer to still active star formation regions.
\end{abstract}

\section{Introduction}

We report the preliminary results of an investigation of the relation of the $\mathrm{Li}$ abundance in $\mathrm{T}$ Tauri stars with their distance to the nearest star-forming region. The lithium abundance is potentially an age indicator; it is believed that the youngest $\mathrm{T}$ Tauri stars present the same $\mathrm{Li}$ abundance of the interstellar medium, and that the surface abundance gradually decreases with time, due to the combined effect of convection and destruction in the stellar interior (Spite and Spite, 1982; Duncan and Jones, 1983). It would be of great interest to be able to estimate the age of $T$ Tauri stars, in order to study their evolution as well as the evolution of the star-forming regions.

\section{Observational data}

Our program stars have been observed in a recent survey for new $T$ Tauri stars (TTS) based on the IRAS point source catalog (Gregorio-Hetem et al.,1991). In the part already completed of the survey, we obtained Coudé spectra of 62 TTS (including previously known TTS), with the $1.6 \mathrm{~m}$ telescope of the Laboratorio Nacional de Astrofísica, Brazópolis, Minas Gerais. The observed spectral region is 6550-6750 $\mathrm{A}$, which contains $\mathrm{H} \alpha$ and the $\mathrm{Li}$ resonance line at $6707 \mathrm{~A}$; the resolution is $0.1 \mathrm{~A}$. We also made UBVRI photometric measurements of most of the program stars with a $0.6 \mathrm{~m}$ telescope at the same observatory.

\section{Temperature and $\mathrm{Li}$ abundance determinations}

Since the surface lithium abundance is known to be a function of both age and mass (or temperature), we determined the temperatures of the stars of our sample by fitting, with a model, our photometric data and the IRAS data. The model consists of a central star, represented by a blackbody, surrounded by a spherically symmetric circumstellar dust shell (CDS). Good fits are obtained by taking CDS density decreasing like $r^{-1.5}$ and temperature decreasing like $r^{-0.4}$, and an extinction law like $\lambda^{-1}$. The internal radius of the CDS is fixed by considering that the radiative equilibrium temperature of the dust grains cannot exceed typical evaporation temperature of about $400 \mathrm{~K}$. In some cases an optically thick, geometrically thin disk with temperature decreasing outwards like $r^{-0.75}$ is needed to obtain a good fit. The stellar temperatures obtained in this way are in general in good agreement with the spectral types, when they are known.

Lithium abundances were estimated by comparing the stellar temperature and the equivalent width of the Li I $\lambda 6707$ line with a grid of curves of growth presented by Duncan (1991), from Kurucz and Bell and Gustafsson model atmospheres, for $\log g=4.0$ and $\log g=3.75$. 


\section{Results and discussion}

We present in Figure $1 \log \mathrm{Li}$ abundance as a function of $\log \mathrm{T}$. The well known depletion of $\mathrm{Li}$ in low temperature TTS can be observed. We remark that we obtain, for many TTS with T $>5000 \mathrm{~K}$, abundances of the order of $\log N(\mathrm{Li})=4$, on the $\log \mathrm{N}(\mathrm{H})=12$ scale. This is much larger than the accepted primordial abundance of $\mathrm{Li}, \log \mathrm{N}(\mathrm{Li})<3$ (Boesgard and Steigman, 1985). One possibility would be to suspect that the model atmospheres are not correct. Another possibility would be a segregation mechanism to act during the last stages of formation of stars, if for instance $\mathrm{Li}$ is tied to dust grains submitted to radiation pressure, so that its accretion on the star is delayed, resulting in a relative enrichment of the photosphere.

We present in Figure $2 \log$ of $\mathrm{Li}$ abundance versus distance of the TTS to the nearest dense core of a star forming molecular cloud. The distances are projected distances measured in degrees; the reason for the use of angular units is that the distances of most of the relatively isolated TTS are not known. At $150 \mathrm{pc}$, which is the approximate distance of nearby star-forming cloud complexes like $\rho$ Oph and Cham I, one degree correspond to about 3 pc. Most TTS are situated within 2 degrees of a molecular cloud core; up to such separation they can be considered as belonging to the same association. The relative position, in galactic coordinates, of the TTSs studied here and of the cloud complexes nearest to them are illustrated in Figure 3. For the stars situated at $d>2^{\circ}$, we observe in Figure 2 = tendency of $\mathrm{Li}$ abundance to decrease with $d$.

The obvious interpretation is that this is an effect of age, the more isolated TTS being the oldest ones. Since we do not observe any systematic variation of temperature with distance, we can eliminate the possibility of the more distant TTS being less massive stars. This could be the case if the more distant stars were objects ejected from star-forming regions with larger velocities. Such a mass-selection effect can probably only be observed if stars of a same association (within $2^{\circ}$ ) are compared.

From the $\log \mathrm{N}(\mathrm{Li})$ versus $\mathrm{T}$ and versus age relation presented by Basri, Martin and Bertout (1991), we conclude that Hen 1, the TTS with highest galactic latitude discovered in our survey, is $5^{*} 10^{7}$ years old, and that the group of TTS situated around the "isolated" TTS TW Hya, is about $3^{*} 10^{7}$ years old.

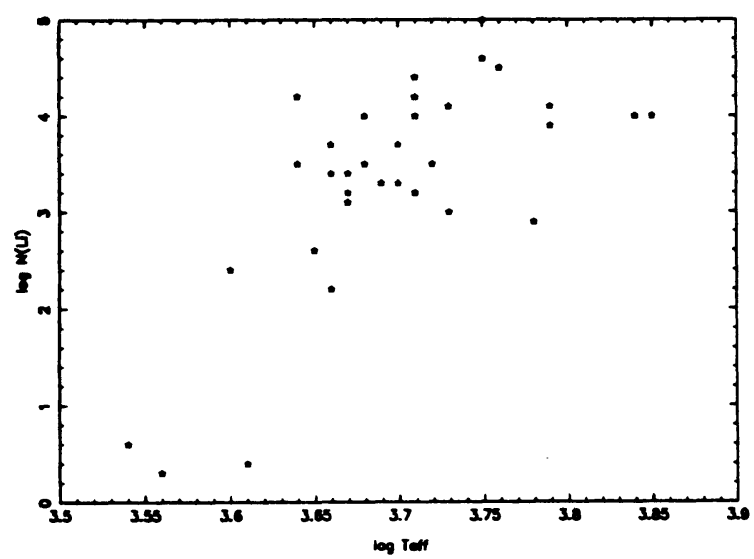

Figure 1: log of Lithinm ebundance versus log of effective temperature of $T$ Tauri stars. 


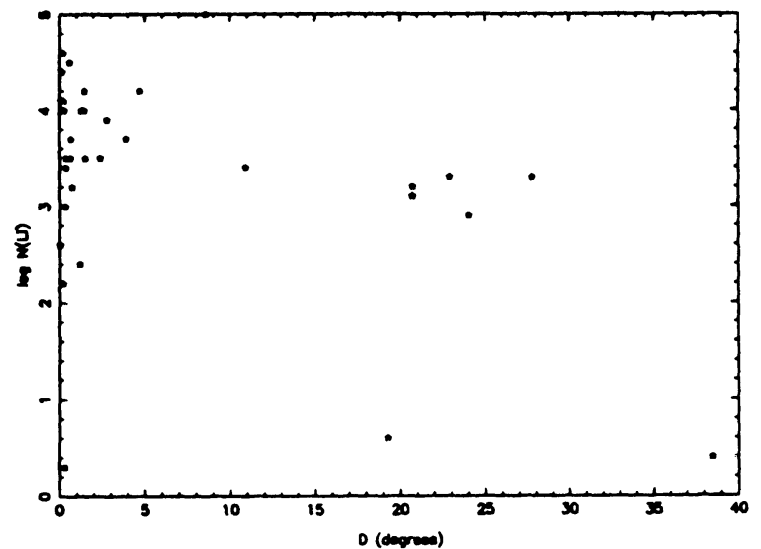

Figure 2: log of Lithium abuadance veruus projected distances to the neareat dence core of a otar forming moleculer cloud.

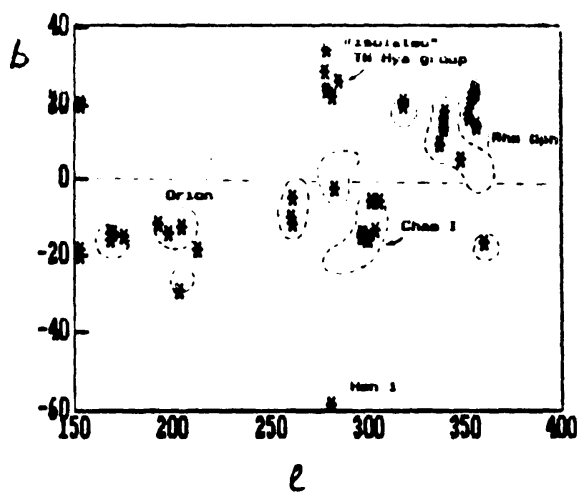

Figuse s: The spatial diatribution of the $\mathbf{T}$ Thuri stare of out sample, in galactic coordinates. Some of the mont important atar-formation regions are indicated.

\section{References}

Basri,G., Martin,E.L., Bertout,C, 1991: A \& A, in press

Boesgard, A.M., Steigman,G.: 1985, ARA \& A 23,319

Duncan, D.K.: 1991, Ap.J, in press

Duncan, D.K., Jones,B.F., 1983, Astrophys. J. 271:66

Gregorio-Hetem,J., Lépine, J.R.D., Torres, C.A.O, Quast, G.R.,

de la Resa, R.: 1992, A J., in press

Spite,F., Spite, M.: 1982, A \& A 115, 357 